\title{
Blockchain's Practical and Legal Implications for Global Trade and Global Trade Law
}

\author{
Emmanuelle Ganne*
}

\section{A INTRODUCTION}

Technology is not only transforming international trade, it is also pushing the boundaries of regulation. The cross-border nature of the Internet challenged existing regulatory approaches, raised new regulatory issues and gave rise to new forms of governance. Digital technologies that leverage the Internet are challenging existing approaches even further. Among those, one technology, blockchain, keeps making the headlines. A game changer for some, the most overhyped technology for others. Few technologies have sparked so much debate.

Often associated with Bitcoin because it was first implemented as the technology underpinning the famous cryptocurrency, blockchain is much more than Bitcoin. ${ }^{1}$ In fact, by making it possible for actors along the supply chain to interact on a peerto-peer basis in quasi real time and in a highly secure and trusted environment, this technology could have a major impact on many facets of international trade and deeply transform it. Blockchain could facilitate international trade transactions and help implement World Trade Organization (WTO) agreements, and it could foster the digitalization of trade.

Yet, technology is only a tool. A number of regulatory issues deserve the attention of policymakers for this potential to be realized. It is therefore critical that government officials educate themselves to understand the technology, its potential, but also its limitations, and keep an eye on developments. This chapter discusses the measures that should be taken to promote the development of a regulatory

* Emmanuelle Ganne is a Senior Analyst at the World Trade Organization. Contact: emmanuelle.ganne@wto.org. The opinions expressed in this chapter are those of the author. They are not intended to represent the positions or opinions of the WTO or its members and are without prejudice to members' rights and obligations under the WTO. Any errors are attributable to the author.

1 This chapter focuses on the technology itself, not on cryptocurrency applications. 
framework conducive to the development of the technology and the role that the WTO could play in this respect.

The chapter's first section describes blockchain's key features and discusses how this technology can be used to facilitate transactions in various areas of global trade and help implement WTO agreements. It examines the potential impact of this technology on international trade. The second section looks at discrete regulatory issues that deserve the attention of regulators for blockchain to truly transform international trade. The last section discusses measures that should be taken to promote the development of a regulatory framework conducive to the development of the technology and the role that the WTO could play in this respect. The chapter argues that the WTO is uniquely positioned to play a pivotal role in ensuring that a conducive governance framework is put in place to allow blockchain to be used to its full potential in the area of international trade.

\section{B UNDERSTANDING BLOCKCHAIN'S PRACTICAL IMPLICATIONS FOR INTERNATIONAL TRADE}

In spite of the many headlines on blockchain, the technology, its functioning and potential to transform business beyond the world of cryptocurrencies and of finance more generally remains difficult for many to apprehend. This section seeks to provide a basic understanding of how the technology works and discusses its practical implications for international trade and the implementation of WTO agreements.

\section{Blockchain: A Complex World}

The catchy word of blockchain conceals a complex reality. The term blockchain is now often used in a generic way to refer to distributed ledger technology (DLT) and this chapter follows this practice. Strictly speaking, however, blockchain is only one type of DLT - one that combines transactions in blocks and links them in a linear way. While there are many different types of DLTs, all possess a number of key characteristics that render them particularly useful as a facilitator of a wide range of international trade processes.

A blockchain, or distributed ledger, is a shared and synchronized digital database that is maintained by an algorithm and stored on multiple 'nodes', i.e. computers connected to the network that store a local version of the ledger. Unlike traditional databases, distributed ledgers have no central data store or entity controlling the network. They function on a peer-to-peer basis without the need for the intermediaries who traditionally authenticate transactions. Data added to the ledger are shared with all participants in quasi real time, verified and validated ('mined' in the context of blockchain technology) by anyone with the appropriate permissions on the basis of the consensus protocol of the ledger, and timestamped. Therefore, participants in 
TABLE 6.1. Types of blockchain platforms

\begin{tabular}{|c|c|c|c|c|}
\hline Blockchain types & $\begin{array}{c}\text { Level of } \\
\text { centralization }\end{array}$ & Read & Write & Example \\
\hline $\begin{array}{l}\text { Public } \\
\text { permissionless }\end{array}$ & $\begin{array}{l}\text { Highly } \\
\text { decentralized }\end{array}$ & Anyone & Anyone & Bitcoin \\
\hline $\begin{array}{l}\text { Public } \\
\text { permissioned }\end{array}$ & $\begin{array}{l}\text { Highly } \\
\text { decentralized }\end{array}$ & Anyone & $\begin{array}{l}\text { Authorized } \\
\text { participants }\end{array}$ & Sovrin \\
\hline $\begin{array}{l}\text { Consortium } \\
\text { permissioned }\end{array}$ & $\begin{array}{l}\text { Partially- } \\
\text { decentralized }\end{array}$ & $\begin{array}{l}\text { Authorized } \\
\text { participants }\end{array}$ & $\begin{array}{l}\text { Authorized } \\
\text { participants }\end{array}$ & Tradelens \\
\hline $\begin{array}{l}\text { Private } \\
\text { permissioned }\end{array}$ & Centralized & $\begin{array}{l}\text { Authorized } \\
\text { participants }\end{array}$ & $\begin{array}{l}\text { Authorized } \\
\text { participants }\end{array}$ & $\begin{array}{l}\text { Company } \\
\text { blockchain }\end{array}$ \\
\hline
\end{tabular}

Source: Author.

a distributed ledger have all access to the same information at any time. In other words, a distributed ledger is a shared, trusted record of transactions that all participants can access and check at any time, but that no single party can control (unless it is fully private - see Table 6.1), which allows people with no particular trust in each other to collaborate without relying on trusted intermediaries. Distributed ledgers ensure immediate, across the board transparency.

A distributed ledger is secured using a blend of proven cryptographic techniques. Data entered onto the blockchain are 'hashed', i.e., converted into a new digital string of a fixed length using a mathematical function, and encrypted to ensure data integrity, prevent forgery, and guarantee that the message was created and sent by the claimed sender and was not altered in transit. Records are also linked to one another; attempting to alter the ledger is a difficult endeavour as previous blocks or records of transactions would also have to be altered for the changes to remain undetected. Because of the distributed nature of blockchain, falsifying data or compromising the whole network would require compromising a large number of nodes, which would be practically very hard.

These different characteristics make distributed ledger technology highly secure and difficult to hack - which led The Economist to call blockchain a 'trust machine'.2 They also render DLT a particularly helpful technology to remove frictions from global trade by making it possible for the many stakeholders involved in international trade transactions to interact in a more efficient way.

Distributed ledgers are, to date, the most secure type of databases, ${ }^{3}$ but this is not to say that they are completely immune from tampering or cyberattacks. A distributed ledger can be compromised if a validator or a pool of validators control

2 'The Trust Machine: The Promise of the Blockchain', The Economist, 31 October 2015.

3 However, not all distributed ledgers provide the same level of security. More centralized ledgers are less resilient to outside attacks, and there is a greater risk of human tampering with data. 
more than 50 per cent of the network's computing power, which is called the ' 51 per cent attack'. With computing power capacity of some blockchains being increasingly aggregated, the risks are certainly growing. In fact, in July 2019, two mining pools of the Bitcoin network, one reputed the most difficult to hack, carried out a $5^{1}$ per cent attack on the network in an apparent effort to stop an unknown miner from taking coins that they were not supposed to have access to in the wake of a code change. ${ }^{4}$ While the attack was arguably conducted with a view of doing something good for the community, not to reward the attacker or steal funds, it has led to heated debates in the information technology (IT) community as to the severity of the potential consequences of such attacks. Advances in quantum computing could in the long term also represent a threat to blockchain as blockchain's resilience relies on encryption and algorithms, whose strength is based on computing power. 'Postquantum' algorithms that would be resistant to quantum computing are being actively researched.

\section{A Multitude of Distributed Ledger Technologies}

In spite of these common characteristics and as noted earlier, DLTs are very diverse and there is a multitude of consensus protocols. Consensus protocols govern the way transactions are validated and records are added to the network and differ in terms of energy consumption and rapidity at which blocks or transactions can be validated. Some of the most well-known consensus protocols include proof-of-work (PoW), which is used by Bitcoin; proof-of-stake $(\mathrm{PoS})$, which is being considered by Ethereum, another well-known public blockchain, and Proof of Elapsed Time (PoET) used by Hyperledger Sawtooth. Proof-of-work requires that the participants who validate blocks, the 'miners', show that they have invested significant computing power to solve a hard cryptographic puzzle. Miners compete with each other to validate a block and add it to the blockchain. The miner who validates the new block is rewarded with Bitcoins. The level of difficulty of the mathematical problem increases as blocks are mined to ensure that only one block can be mined every ten minutes. The big disadvantage of proof-of-work is its high level of energy consumption - which researchers estimated to be as high as that of a country like Ireland. ${ }^{5}$ Proof-of-stake algorithms were developed to overcome the disadvantage of PoW in terms of energy consumption. PoS replaces the mining operation with rewards in proportion to the amount of the validators' 'stake' in the network (i.e. ownership or assets of cryptocurrency in the network). As for Proof of Elapsed Time, it uses a

4 One of these two pools is said to have controlled at some point more than 50 per cent of the hashing power on its own. See A. Hertig, 'Bitcoin Cash Miners Undo Attacker's Transactions with “51\% Attack”, available at www.coindesk.com/bitcoin-cash-miners-undo-attackers-transac tions-with-51-attack.

5 K. O'Dwyer and D. Malone, 'Bitcoin Mining and Its Energy Footprint', National University of Ireland Maynooth Working Paper (2014). 
random leader election model, or a lottery-based election, with the protocol randomly selecting the next leader to finalize the block. These are merely a few examples of the many different consensus protocols that exist in practice.

In addition, while the most well-known DLT, blockchain, combines transactions in blocks and chains them in a linear way - hence the term 'blockchain' - an increasing number of models of transaction flows are being developed, which move away from the concept of 'blocks' - or even from both concepts of 'blocks' and 'chain'. The so-called 'New kids not on the blocks' include IOTA, ${ }^{6}$ Ripple$^{7}$ and Hedera Hashgraph. ${ }^{8}$ In IOTA, for example, transactions are not grouped into blocks and each transaction is linked to two previous transactions as part of the validation process to form a 'tangle'.

Despite these important technical issues, the technology itself is only one part of the story and the term 'blockchain' is often used to refer to the platforms that are being developed for specific applications, the nature of which varies greatly. While blockchain was originally envisioned as a decentralized network open to everyone, a number of platforms have emerged that are controlled by a company or a group of companies forming a consortium and whose access is limited to authorized participants.

\section{Various Types of Blockchain Platforms}

Distributed ledgers are often classified as public versus private or 'permissioned' versus 'permissionless'. Under the category of private blockchain or ledger, there is a subtype called 'consortium' that is sometimes considered as a type of blockchain in its own right. 9 These two classifications are at times conflated and it is not uncommon for people to associate public with permissionless and private/consortium platforms with permissioned platforms. The reality is, however, slightly more complicated, as some public platforms can be permissioned (see Table 6.1). ${ }^{10}$

In essence, a permissionless blockchain is a platform that is open to anyone, with no restrictions imposed on who can access the platform and validate transactions, while a permissioned blockchain is a platform in which access is restricted. The distinction between public, consortium and private blockchains is linked to the degree of decentralization. A public platform is a platform that is highly decentralized, with no specific entity/entities managing the platform. Transactions are public and individual users can maintain anonymity and no user is given special privileges

\footnotetext{
www.iota.org.

https://ripple.com.

www.hedera.com. For details, see E. Ganne, Can Blockchain Revolutionize International Trade? (Geneva: WTO, 2018).

9 V. Buterin, 'On Public and Private Blockchains', Ethereum Blog, 7 August 2015, available at https://blog.ethereum.org/2015/08/o7/on-public-and-private-blockchains/.

10 Ganne, note 8.
} 
over any decision. In contrast, in a private blockchain, the permissions to validate and add data to the ledger are controlled by one entity that is highly trusted by the other users, and participants are identified. The term 'blockchain' in the context of private ledgers is controversial and disputed, as such highly centralized ledgers have little in common with the original idea behind blockchain. A consortium blockchain is a 'partially decentralized' platform ${ }^{11}$ that operates under the leadership of a group rather than a single entity and in which participants are identified. One of the distributed ledger technologies often used for private or consortium platforms is Hyperledger Fabric, ${ }^{12}$ which was developed by IBM, and donated to the Hyperledger Project of the Linux Foundation, and has been designed to cater to the needs of participating companies.

Private and consortium platforms provide for greater scalability but at the expense of decentralization. Public platforms, on their side, are highly decentralized and provide for a high level of security, but this comes at the cost of efficiency and scalability. This is what Vitalik Buterin, founder of Ethereum, called the blockchain trilemma' - i.e., the impossibility to achieve scalability, decentralization and security simultaneously in a blockchain. At most, two of these properties can be achieved. Other researchers articulate the trilemma around a slightly different set of concepts: decentralization, correctness and cost efficiency, ${ }^{13}$ but the conclusion remains the same: you cannot have it all.

\section{Automation via Smart Contracts}

A particularly interesting feature of the blockchain universe is the possibility to use smart contracts, i.e. computer programmes that automatically enforce themselves (self-execute) without the intervention of a third party when specific conditions are met (based on the 'if ... then...' logic; e.g., if the goods are unloaded at port of X, then funds are transferred). Smart contracts state the obligations of each party to the 'contract', as well as the benefits and penalties that may be due to either party under different circumstances. However, unlike the name suggests, smart contracts are neither smart, as there is no cognitive or artificial intelligence component to them, nor are they contracts in a legal sense.

Smart contracts go back many years. Cryptographer Nick Szabo introduced them first in various publications during $1994-1997,{ }^{14}$ but their use outside of blockchain

${ }^{11}$ Buterin, note 9.

12 www.hyperledger.org/projects/fabric.

${ }^{13}$ J. Abadi and M. Brunnermeier, 'Blockchain Economics', Princeton University Working Paper (2018).

${ }^{14}$ Nick Szabo defined smart contracts as 'a set of promises, specified in digital form, including protocols within which the parties perform on the other promises.' The general objectives of smart contract design are to satisfy common contractual conditions (such as payment terms, liens, confidentiality and even enforcement), minimize exceptions both malicious and accidental, and minimize the need for trusted intermediaries'. See N. Szabo, 'Smart Contracts: 
makes them subject to the same problems as centralized databases - that is, a single point of failure and the possibility to change the data easily. When used in the context of blockchain they inherit blockchain's key properties, such as immutability.

\section{Blockchain: A Potentially Transformative Impact on International Trade}

The transparent, highly secure and quasi-immutable nature of blockchain makes it an interesting tool to facilitate a number of processes related to international trade. A myriad of proofs of concepts and pilot projects leveraging the technology have been developed in virtually all areas of international trade, from trade finance to border procedures and the management and enforcement of intellectual property rights, to cut costs, streamline procedures, and help move away from heavy paper-based processes, with an increasing number of projects now entering the production phase. The potential of this technology to transform international trade is indeed significant. ${ }^{15}$

\section{Blockchain's Potential Impact on International Trade Transactions}

International trade has seen little innovation since the invention of the container by Malcolm McLean in 1955. Goods are still transported across oceans in the same old way, requiring paper and labour-intensive processes. In a now well-known experiment, shipping company Maersk followed a container of roses and avocadoes from Mombasa in Kenya to Rotterdam in the Netherlands in 2014 to document the maze of physical processes and paperwork that impact every shipment. Around 30 actors and more than 100 people were involved throughout the journey, leading to more than 200 interactions. ${ }^{16}$ The shipment generated a pile of paper $25 \mathrm{~cm}$ high and the cost of handling it was higher than the cost of moving the container. ${ }^{17}$ One of the critical documents went missing, only to be found later amid a pile of paper. ${ }^{18}$ The system is overall slow, costly and inefficient. The use of blockchain opens incredible opportunities to cut costs and improve processes, and to truly digitize procedures that are still analogue.

A BLOCKCHAIN CAN MAKE TRADE PROCESSES MORE EFFICIENT AND LESS COSTLY Because it allows all actors to interact in real time in a highly secure

Building Blocks for Digital Markets', Extropy: The Journal of Transhumanist Thought 16 (1996), 50-53, at 51. Available at: https://archive.org/details/extropy-16/page/5o/mode/2up?q=a+computerized+protocol+that+executes+terms

15 Ganne, note 8.

${ }^{16}$ F. Landon, 'Maersk, Avocados and the Global Trade Paperchase', SeaTrade Maritime News, 29 November 2017.

${ }^{17}$ I. Allison, 'Shipping Giant Maersk Tests Blockchain-Powered Bill of Lading', International Business Times, 14 October 2016.

${ }^{18}$ K. Park, 'Blockchain Is about to Revolutionize the Shipping Industry', Bloomberg, 18 April 2018. 
environment, blockchain can make processes more efficient and less costly. Once added to the ledger, information is available to all participants simultaneously, and the nature of the technology gives participants the guarantee that the information cannot be tampered with, thereby generating trust.

In one of the first economic studies on blockchain, Catalini and Gans consider that the use of blockchain affects two key costs in particular: (i) verification costs, i.e. the ability to verify the attributes of a transaction cheaply and (ii) networking costs, i.e., the ability to bootstrap and operate a marketplace without the need for a traditional intermediary. ${ }^{19}$ Other costs, such as coordination and processing costs, financial intermediation and costs related to foreign exchange could be affected as well. ${ }^{20}$ While the potential impact on trade costs has not been thoroughly researched yet, various studies by actors in the field estimate that the potential savings from full digitalization using blockchain could represent between 15 and 30 per cent of the costs of the processes concerned. ${ }^{21}$ The reduction in trade costs can be particularly interesting for small- and medium-sized enterprises (SMEs) who face higher fixed costs than large companies.

The potential efficiency gains of blockchain have led many actors involved in international trade to build consortia to leverage the opportunities that the technology opens. IBM and Maersk were the first ones to open the race with their platform Tradelens ${ }^{22}$ that aims to connect the various parties involved in international trade from freight forwarders to government authorities and banks - and to digitize the supply chain from end to end, with a view to streamlining and facilitating procedures. The platform is now fully operational and claims to process ten million events a week. Others are following suit.

Numerous initiatives have also been launched in the area of trade finance: Contour, Komgo, We.trade, eTradeConnect are some of the bank-led projects that aim to address deficiencies of trade finance processes using distributed ledger technology. Traditional trade finance, in particular letter of credit transactions, is labour and paper intensive and involves multiple players, generating much inefficiency. Research by the Boston Consulting Group found that more than twenty players are usually party to a single trade finance transaction throughout the process, with data captured in ten to twenty documents, creating approximately five thousand data field interactions, but that only 1 per cent of these interactions creates value. The remaining 85-90 per cent of the transactions simply consist of ignore/

19 C. Catalini and J. S. Gans, 'Some Simple Economics of the Blockchain', MIT Sloan Research Paper No 5191-16 (2019).

2o Ganne, note 8.

${ }^{21}$ Accenture, Banking on Blockchain - A Value Analysis for Investment Banks (New York/ London: Accenture, 2017); I. Allison, 'Maersk and IBM Want ten Million Shipping Containers on the Global Supply Blockchain by Year-End', International Business Times, 8 March 2017.

22 www.tradelens.com/. 
transmit to the next party' actions. ${ }^{23}$ Not surprisingly, banks see blockchain as a potential tool to reduce coordination costs between the multiple actors involved in a letter of credit transaction. The first results of initiatives using DLT to process letters of credit seem encouraging, arguably reducing the time needed to process letter of credit transactions from on average of five to ten days to a matter of hours. ${ }^{24}$

Yet, all these projects are still in their early stages, being at best a two or three of years. It remains to be seen whether these various platforms will effectively generate the expected outcomes and manage to scale up to become viable business projects.

B TOWARDS PAPERLESS TRADE? Efforts to digitize trade have so far been impeded by what is usually referred to in the blockchain world as the 'double-spend problem', i.e. the possibility to spend a digital asset twice, which translates in the non-currency world as the possibility to make multiple copies of digital files. This is particularly important in the case of international trade, as a document like the bill of lading represents ownership of the goods. It is critical to ensure that an electronic bill of lading can be transferred from one holder to another in a manner that guarantees that there is only one holder at any moment in time and that multiple copies cannot be put in circulation. Simple digitization through PDFs, for example, does not provide these assurances. However, blockchain does. Not only does it provide the guarantee that there exists only one copy of the document, but it also allows tracing the transfer of the file along the journey. In 2018, Accenture completed a proof of concept to digitize bills of lading in cooperation with APL Ltd. (owned by the world's third largest container line), the logistics company Kuehne + Nagel, and Danish customs. ${ }^{25}$ The proof of concept arguably led to an 80 per cent reduction in efforts associated with managing data related to the bill of lading. ${ }^{26}$ While these numbers are difficult to check, the key characteristics of blockchain make it a potentially interesting tool to solve some of the problems associated with electronic bills of lading.

${ }_{23}$ Boston Consulting Group and Swift, 'Digital Innovation in Trade Finance - Have We Reached a Tipping Point?', 19 October 2017, available at www.swift.com/news-events/news/ digital-innovation-in-trade-finance-have-we-reached-a-tipping-point.

24 In a proof of concept carried out in 2016, Barclays and fintech startup Wave completed a letter of credit transaction for a shipment of cheese and butter from Ireland to the Seychelles in less than four hours while it usually takes about ten days. See Barclays, "The Blockchain Revolution in Trade Finance', 30 September 2016, available at www.barclayscorporate.com/insights/innov ation/blockchain-revolution-in-trade-finance/. In May 2018, HSBC completed a live letter of credit operation, reducing the time needed to process the transaction to around twenty-four hours. See D. Weinland, 'HSBC Claims First Trade-Finance Deal with Blockchain', The Financial Times, 13 May 2018.

25 'APL Tests Blockchain Solution', The Maritime Executive, 17 March 2018, available at www .maritime-executive.com/article/apl-tests-blockchain-solution.

${ }^{26}$ Author's interview with Accenture in 2018. 
Before the advent of blockchain, digitization efforts of companies like essDocs and Bolero mainly focused on digitizing payments and information, essentially via scanned PDF documents. They did little, however, to digitize the transactions themselves. ${ }^{27}$ By allowing participants in the network to interact in real time in a highly secure environment, blockchain opens the door to the true digitization of transactions. While the rise of the Internet had a profound impact on the way we communicate, blockchain has the potential to impact transactions. Sometimes called the 'Internet of value', blockchain and distributed ledger technologies are best described in my view as the 'Internet of transactions'. By breaking the various silos that currently exist between the many parties involved in cross-border trade transactions, blockchain could give rise to a 'global asset web' and bring trade globalization to another level.

Beyond these generic considerations on the potential impact of blockchain on international trade, this technology can prove particularly useful for the implementation of the various WTO agreements, as will be explained in the following section.

\section{Blockchain Can Help Implement WTO Agreements ${ }^{28}$}

Blockchain could help implement various provisions of the recently adopted Trade Facilitation Agreement (TFA). In particular, it could prove useful to enhance interagency cooperation, as it allows all participants to interact directly and in quasi real time (Article 8 TFA). ${ }^{29}$ It could improve the efficiency of customs clearance processes and reduce the need for manual verification. Requests for advance rulings (Article 3 TFA), if submitted through a blockchain platform, would be securely stored on the blockchain, in a permissioned ledger, and remain accessible at all times by authorized stakeholders, including all customs offices located in the territory, throughout the validity period of the ruling, thereby facilitating the release and clearance process. The sharing of required data on the ledger in real time could facilitate pre-arrival processing and expedited release of goods (Article 7.1 and 7.8 TFA). The use of smart contract could help optimize risk management (Article 7.4 TFA) - customs documents submitted via the system would be immediately and automatically analyzed and assessed on the basis of pre-determined selectivity criteria encoded in a smart contract - and post clearance audit (Article 7.5 TFA), the tamper-proof nature of the technology making it possible to easily track and audit transactions. Blockchain could also help handle temporary admission of goods processes (Article 10.9 TFA). It has also been argued that blockchain could help

27 H. Castell, 'Blockchain in Trade: Are We Missing the Point?', TXF News, 8 January 2018.

28 This section focuses on multilateral WTO agreements. It is worth noting, however, that DLT can also prove interesting in the context of government procurement. See Ganne, note 8.

29 World Customs Organization, 'Blockchains', Information Management Sub-Committee, $72 n d$ Meeting, 19 April 2017. 
administer single windows in a more efficient way (Article 10.4 TFA). ${ }^{30}$ Finally, blockchain could facilitate revenue collection through the use of smart contracts and the management of authorized operators status (Article 7 TFA).

In fact, the potential of the technology to facilitate these processes is already being tested. The European Commission carried out a successful proof-of-concept in cooperation with the International Chamber of Commerce (ICC) related to ATA-carnets used for the temporary admission of goods. ${ }^{31}$ The Republic of Korea's customs authority is working with e-commerce companies to leverage the technology to accelerate customs clearance of e-commerce goods from these companies, share information in real time, generate automated import customs clearance report to authorities, and prevent fraud and smuggling. ${ }^{32}$ A project called Cadena is also underway between Mexico, Peru and Costa Rica with the support of the Inter-American Development Bank to create a common platform for the management of authorized operators (or authorized economic operators, AEOs). Cadena aims to automate the process of sharing AEO data among the parties and remedy some of the problems faced in the implementation of AEO mutual recognition agreements. The problematic areas include manual processes of sharing sensitive and/or confidential data with low standards of security and integrity; the difficulty to establish the provenance and traceability of the data and to guarantee secure access; the inability to grant $\mathrm{AEO}$ benefits in real time; and the inability to react in real time when a suspension occurs, with all the consequences that this may have on the security of the supply chain. ${ }^{33}$

Blockchain could also help implement the Import Licensing Agreement in a more efficient way, in particular the provisions on application for import licenses (Article 1.6) and automatic import licensing (Article 2). This information, once added to the ledger, would be directly accessible to all relevant stakeholders thereby limiting the number of agencies to approach - and the use of smart contracts could automate the granting of licenses. It could also help administer import and export licenses. Such licenses are normally delivered for a set period of time. Storing an import or export licence on a blockchain platform would save the importer or exporter the trouble of having to keep the licence in a safe place to avoid losing it and would allow customs authorities to easily check the authenticity and validity of the permit. ${ }^{34}$ Using fake permits would no longer be

$3^{\circ}$ Ganne, note 8; Inter-American Development Bank and World Economic Forum, 'Windows of Opportunity: Facilitating Trade with Blockchain Technology', White Paper, July 2019.

${ }^{11}$ Z. Saadaoui, 'Digitization of ATA Carnets: How the Blockchain Could Enhance Trust', WCO Magazine, 2018.

32 S. Das, 'Korea Customs Service to Pilot Blockchain-Based Import Customs Platform', CCN, 6 June 2018, available at www.ccn.com/korea-customs-service-blockchain-customs-clearanceplatform/.

33 S. Corcuera-Santamaria, 'Blockchain Platform to Implement MRAs for AEO Programs', IV AEO Global Conference Kampala, Uganda, 14-16 March 2018.

34 Ganne, note 8. 
possible. ${ }^{35}$ The use of a smart contract could even allow the parties to go one step further by automatically rendering an import/export licence invalid upon expiration of its validity period. This could help fight fraud and avoid situations, as the one with the Philippines in 2016, when the Department of Agriculture cancelled and recalled all import permits on meat products to tackle meat import fraud, having found that old permits were being recycled to smuggle imports. ${ }^{36}$

In the context of the Technical Barriers to Trade (TBT) and Sanitary and Phytosanitary Measures (SPS) agreements, the traceability and transparency features of blockchain can prove interesting to help assess sanitary risks (Article 5 SPS Agreement), prove conformity assessment and manage conformity assessment procedures (Article 5 TBT Agreement), and demonstrate compliance with standards. While traditional labelling systems can be easily manipulated, blockchain provides a highly secure system to prove key characteristics of the products concerned. Numerous start-ups and well-established companies, such as Provenance, ${ }^{37}$ Verified Organic ${ }^{38}$ or Bext $360^{39}$ are turning to blockchain to assert ethical, organic or quality claims. The use of blockchain is also being explored for the granting of ephyto-certificates to help streamline the approval workflow of such certificates. ${ }^{\circ}$

Blockchain could facilitate assessment of origin, be it for the purposes of the WTO Agreement on Rules of Origin that applies to non-preferential rules of origin or for the purposes of a preferential trade agreement between two or more parties. Various companies, such as EssDocs ${ }^{41}$ and VCargoCloud in Singapore, ${ }^{42}$ as well as chambers of commerce in Singapore and Dubai, are testing the technology in relation to certificates of origin. ${ }^{43}$ If blockchain traceability from farm or factory to

35 In December 2017, the National Food Authority (NFA) of the Philippines issued a warning against individuals or entities using fake or fabricated rice import permits following a report that some unscrupulous individuals or parties were selling spurious permits allegedly issued by the NFA under the 2017 minimum access volume private sector rice import scheme. See www.nfa .gov.ph/35-news/1053-nfa-warns-against-fake-rice-import-permits.

$3^{6}$ A. Fortune, 'Philippines Takes on Meat Import Fraud through Permit Recall', GlobalMeat News, 23 November 2016.

37 www.provenance.org/.

$3^{8}$ www.verifiedorganic.io/.

39 www.bext360.com/.

$4^{\circ}$ 'Antwerp Blockchain Pilot Pioneers with Secure and Efficient Document Workflow', Port of Antwerp, 18 June 2018, available at www.portofantwerp.com/en/news/antwerp-blockchain-pilotpioneers-secure-and-efficient-document-workflow.

${ }^{41}$ essDOCS, 'Introducing essCert - A Next Generation eCO Solution', essDOCS News, 31 May 2018, available at: www.essdocs.com/blog/introducing-esscert-next-generation-eco-solution.

42 www.vcargocloud.com/.

43 An important point to note when it comes to certificates of origin is that authentication from chambers of commerce does not attest to the true origin of the product, only to the statement provided to the chambers of commerce by the exporter, leading some to argue that such authentication would, in reality, not be truly necessary. Blockchain would not change this state of affairs. Arguably, the benefits of a blockchain-based system when issuing certificates of origin would be limited to proving that the certificate is authentic - i.e. that it has been delivered by the pertinent authority - and has not been tampered with (Ganne, note 8). 
shelf becomes more widely used, the determination of origin could become much easier. One could even imagine a day when certification of origin would rely on blockchain data to be determined directly at the border, without the need for a certificate or origin - provided the systems put into place are accessible by customs authorities and not confined to the internal supply chain of companies.

Another area where blockchain could have a significant impact is intellectual property (IP). Beyond blockchain's potential to provide proof of existence and ownership and to ease registration of IP rights, ${ }^{44}$ which are issues of great importance to right holders but not directly relevant in the context of the WTO, as it is the World Intellectual Property Organization (WIPO) that administers the relevant treaties, blockchain can facilitate the implementation of various provisions of the Agreement on Trade-Related Aspects of Intellectual Property Rights (TRIPS). In particular with regard to Articles 51 and 52 TRIPS, blockchain can be of help. Article 51 requests members to put in place procedures to enable right holders to request the suspension by the customs authorities of the release into free circulation of goods that they suspect infringe IP rights. Right holders initiating such procedures must, under Article 52, provide adequate evidence that there is prima facie infringement of the right holder's right. In the same spirit, Article 58 gives WTO members the possibility to authorize customs officials to act upon their own initiative, ex officio, to suspend the release of goods for which there is evidence that IP rights are being infringed.

The difficulty is proving prima facie evidence of infringement. Most customs officials lack expertise in detecting counterfeit goods. Blockchain, when used in combination with $\mathrm{QR}$ codes or chips embedded in products to trace provenance, can offer an interesting tool to demonstrate prima facie evidence of infringement. If a brand uses blockchain to record the history of its products, the absence of a tag or an incorrect tag on the product would make it easier for the right holder to provide adequate evidence of infringement and for enforcement officers to detect counterfeits. ${ }^{45}$ Various start-ups, such as Provenance, Blockpharma, Blockverify, VeChain and Seal, to name just a few, already offer blockchain-based solutions to help companies producing luxury or fashion products, as well as pharmaceuticals and electronics, fight counterfeit.

Management of IP rights has also been a subject of discussions at the WTO General Council. In December 2016, Brazil submitted a communication calling for a decision on the management of copyright towards fair payment for authors and performers' in which WTO members would 'stress the importance of transparency in the remuneration of copyright and related rights in the digital environment'. ${ }^{6}$ In

44 Ganne, note 8.

45 R. Burstall and B. Clark, 'Blockchain, IP, and the Fashion Industry', Managing Intellectual Property, 23 March 2017.

$4^{6}$ WTO, Electronic Commerce and Copyright, Submission by Brazil, JOB/GC/113, 15 December 2016. 
a follow-up submission circulated in September 2018, Brazil and Argentina noted that information technology could and should facilitate access to real-time data on the use and remuneration of right holders'. ${ }^{47}$ Fair remuneration is a particularly acute problem for authors and performers, who often struggle to be paid for their creation, or when they do, often see a large part of their revenue captured by intermediaries, such as record companies, performance rights organizations and streaming digital service providers like Spotify, in the case of music. Blockchainenabled contracts attached to a creation could allow 'smart management' of IP rights, enabling authors and performers to be paid upon use of their work. UK pop singer Imogen Heap showed the way in 2017 by attaching a smart contract to two of her songs to automatize payments of royalties. She is now working on the creation of a Creative Passport to 'help musicians make money again'. ${ }^{8}$

As this quick overview of blockchain's potential to digitize trade transactions and make trade processes more efficient shows, blockchain's impact on international trade is likely to be wide-ranging and significant. However and as mentioned at the outset of this chapter, technology is only a tool. Without a regulatory environment conducive to its large-scale deployment, the opportunities that blockchain opens to make international trade more efficient could remain unrealized. The next section looks at various regulatory issues that deserve policymakers' particular attention if blockchain is to realize its full potential.

\section{REGULATORY CONSIDERATIONS AROUND BLOCKCHAIN}

Code needs law for recognition, and ultimately, for large-scale adoption. Legal recognition and compliance with existing legal systems is required if blockchain and blockchain-based applications are to be accepted by users as a way to transact with one another and are to have a real value and real-world impact.

Since blockchain belongs to a large category of digital technologies, some of the regulatory issues that it raises are common to other digital technologies - such as for instance the importance of ensuring free data flows. ${ }^{49}$ The key characteristics of blockchain, especially its quasi immutability, the ability to use smart contracts, and the possibility for users to have control over their data, opens new opportunities. But they also give rise to specific regulatory issues that deserve particular attention. This section focuses on such issues in the context of legal recognition of e-signatures, e-documents and blockchain transactions; applicable law, liability and enforcement; as well as data localization and data privacy.

47 WTO, Electronic Commerce and Copyright, Submission by Brazil and Argentina, JOB/GC/ 200/Rev.1, 24 September 2018.

$4^{8}$ I. Heap, 'Blockchain Could Help Musicians Make Money Again', Harvard Business Review, 5 June 2017.

49 See Chapter 1 in this volume. 


\section{Legal Recognition of E-Signatures, E-Documents and Blockchain Transactions}

The large-scale deployment of blockchain requires more than the technology. It requires frameworks that, among other things, recognize e-signatures and e-documents, and clarify the legal status of blockchain transactions..$^{\circ}$ As earlier noted, blockchain has the potential to accelerate the digitalization of trade and to help move towards truly paperless trade. However, full digitization can only become reality if legislation provides for e-authentication methods and for the recognition of esignatures, e-documents and e-transactions. The adoption of the Model Law on Electronic Signatures in 2001 and the Convention on the Use of Electronic Communications in International Contracts in 2005 - both developed by the United Nations Commission on International Trade Law (UNCITRAL) - was a first step. However, only a limited number of countries have legal provisions for such recognitions: the former treaty has been enacted by thirty-two states, while the latter by eleven states only. Even in countries that provide for such recognitions, commercial buyers, importers or authorities often continue to request paper copies. In many other countries, national legislation has to be adjusted to authorize the access and sharing of information with another administration, even at the national level. ${ }^{51}$ The issue of recognition of e-signatures and e-documents is being discussed at the WTO in the context of the WTO Joint Statement on Electronic Commerce that was launched at the Buenos Aires Ministerial Conference in December 2017 and the importance of the issue has been reaffirmed by a series of initiatives in 2019 and 2020 .

An important development was the adoption of the UNCITRAL Model Law on Electronic Transferable Records on 13 July $2017,{ }^{52}$ which enables the use of electronic transferable records and sets out the conditions that must be met if an electronic record is to be treated as a transferable document, i.e., a document that entitles the holder to claim fulfilment of the obligation indicated in the document, such as in the case of bills of lading. The principle of neutrality embodied in the Model Law allows the use of all methods and technologies, including distributed ledger technology, to be accommodated. ${ }^{53}$ If transposed into national legislation, this text could open the way to the legal use of blockchain for international trade transactions. To date, however, only three jurisdications have enacted it, ${ }^{54}$ and there

50 Ganne, note 8 .

51 Ibid.

${ }^{2}$ United Nations Information Service (UNIS), 'UN Commission on International Trade Law Adopts the UNCITRAL Model Law on Electronic Transferable Records', Press Release, 17 July 2017.

53 K. Takahashi, 'Blockchain Technology for Letters of Credit and Escrow Arrangements', Banking Law Journal 135 (2018), 89-103.

54 Bahrain, Singapore and Abu Dhabi Global Market, a recently-created commercial free zone in the heart of the UAE's capital city. 
is still a long way to go towards making this blockchain-enabled environment for transactions real.

Besides general issues related to the legal recognition of e-signatures, e-documents and e-transactions, the legal status of blockchain transactions and smart contracts remains still uncertain, not the least because of a lack of a unanimous definition of the terms 'blockchain' and 'smart contract'. 55 As noted earlier, the term blockchain is often used in its generic sense to refer to DLT but also employed interchangeably to refer to blockchain protocols, services, business applications and platforms, thus creating an unfortunate confusion, especially outside the world of blockchain experts. Initiatives have been launched in various international fora to develop common definitions; work is underway, for instance, at the International Telecommunication Union (ITU) and the International Organization for Standardization (ISO)..$^{56}$

Blockchain transactions also raise classification questions ${ }^{57}$ - for instance: Does information stored on a blockchain platform representing ownership or the existence of an asset prove real ownership or the real existence of that asset? What is the legal status of blockchain registries? ${ }^{58}$ Are existing legal and regulatory frameworks capable of comprehending the growing variety of blockchain applications, concept and use cases? ${ }^{59}$ While smart contracts are not legal contracts per se, to what extent can they be legally binding? ${ }^{60}$ Various governments are now working on or considering legislation to address blockchain and recognize the legal validity of blockchain and blockchain transactions, smart contracts and financial instruments issued on a blockchain platform. In the United States, since 2018, several states have been working on bills to give legal recognition to blockchain transactions, most of them in the form of legislative amendments. The State of Arizona, for example, passed a bill that qualifies blockchain-enabled signatures as valid electronic signatures. ${ }^{61}$ In

55 This chapter focuses on the technology itself, not on cryptocurrencies. One should note, however, that the legal status of cryptocurrencies also varies considerably from country to country. While some countries have explicitly allowed the use of Bitcoin, others have restricted or banned it.

${ }^{56}$ The ITU Telecommunication Standardization Sector established a Focus Group on Application of Distributed Ledger Technology in May 2017 that looks at definition issues and aims to develop a standardization roadmap for interoperable DLT-based services. (See www.itu int/en/ITU-T/focusgroups/dlt/Pages/default.aspx. As for the ISO, it created a committee in 2016 that also looks at definition and standardization issues; see www.iso.org/committee/ 62666o4.html.)

57 K. Werbach 'Trust, but Verify: Why the Blockchain Needs the Law', Berkeley Technology Law Journal 33 (2018), 487-550.

$5^{8}$ R. Herian, Legal Recognition of Blockchain Registries and Smart Contracts (Brussels: EU Blockchain Observatory and Forum, 2018).

59 Ibid.

6o $\mathrm{R}_{3}$ and Norton Rose Fulbright, 'Can Smart Contracts Be Legally Binding Contracts', $\mathrm{R}_{3}$ and Norton Rose Fulbright White Paper, November 2016.

${ }^{61}$ N. De, 'Arizona's Governor Signs Latest Blockchain Bill into Law', CoinDesk, 5 April 2018, available at www.coindesk.com/arizonas-governor-signs-latest-blockchain-bill-into-law. 
Europe, Malta adopted a law in July 2018 to regulate distributed ledger technologies and virtual financial assets, with the goal of promoting Malta as a blockchain island'; ${ }^{62}$ France introduced two bills recognizing blockchain technology in 2016 and 2017. ${ }^{63}$ Private sector initiatives are also exploring ways to make smart contracts more flexible. ${ }^{64}$ Indeed, one of the fundamental reasons often mentioned to argue that smart contracts cannot, as they currently exist, be considered a wholly viable alternative to existing forms of contracts, is their immutability that goes counter to traditional contract law. ${ }^{65}$ Greater flexibility can remedy this.

Overall, despite these various initiatives, there is no coordinated position worldwide on the legal status of blockchain and blockchain-based applications, which gives rise to a risk of regulatory fragmentation that could undermine the deployment of a technology that is built on the premise of breaking silos.

\section{Applicable Law, Liability and Enforcement Issues}

Both permissionless and permissioned blockchain applications raise specific issues in terms of applicable jurisdiction, liability and enforcement, although in slightly different terms. As nodes can be located anywhere in the world, establishing which laws and regulations apply to a given application can be challenging, particularly in the case of public permissionless blockchains. Although one could argue that every transaction falls under the jurisdiction of the location of each participant in the network, the anonymous nature of public permissionless blockchains makes it extremely difficult, if not almost impossible, to identify the processing entity and to pinpoint the place where the contentious transaction was made. The problem is less acute in the case of permissioned blockchains, as participants are known, and the governing law can be determined as part of the governance structure of the blockchain platform. ${ }^{66}$

Blockchain applications also raise issues related to liability and the resolution mechanism in case of conflict, technical problems or unintentional action. While in a private/consortium blockchain, there is clear ownership and responsibility, this

62 A. Alexandre, "Malta Passes Blockchain Bills into Law, "Confirming Malta as the Blockchain Island", Cointelegraph, 5 July 2018, available at https://cointelegraph.com/news/malta-passesblockchain-bills-into-law-confirming-malta-as-the-blockchain-island.

${ }^{6}$ In 2016, France introduced legislative changes to recognize certain mini-bonds issued on blockchains, and in December 2017, it passed a new order to allow for the registration and the transfer of financial securities through distributed ledger technology. See Utilisation d'un dispositif d'enregistrement électronique partagé pour la représentation et la transmission de titres financiers, ordonnance No 2017-1674 (2017).

${ }^{6}$ The ERC1538: Transparent Contract Standard developed by Ethereum designers, for example, seeks to make contract terms ('functions') possible. See N. Mudge, 'ERC1538: Transparent Contract Standard \#1538', GitHub, ${ }_{31}$ October 2018, available at https://github.com/ethereum/ EIPs/issues $/ 1538$.

${ }_{5}$ Herian, note 59; $\mathrm{R}_{3}$ and Norton Rose Fulbright, note 61.

66 Ganne, note 8 . 
is not the case in a public blockchain. Furthermore, the ability to enforce smart contracts via traditional means is limited, not least because it requires the parties to the transaction to be known, which in the case of public permissionless blockchains is challenging. Assuming the parties to a given smart contract are known, the only way to reverse the undesirable outcomes of the coded and executed smart contract would be to create a new smart contract. In the case of permissioned blockchains, rules governing the functioning of the platform, the use of smart contracts and dispute resolution can be established as part of the governance structure of the platform, but the issue remains wide open in the case of public permissionless blockchains.

Specific liability frameworks may also have to be developed to address the needs of certain types of transactions. In the context of international trade, letters of credit, for example, are governed by a specific set of rules developed by the ICC - the Uniform Customs and Practice for Documentary Credits (UCP6oo). In a blockchain system using smart contracts, who would have liability at each stage of the process? Likewise, information required for customs clearance usually has to be submitted by a single declarant, who is liable. In a blockchain system, information can be added by various stakeholders making it impossible to pin down a single declarant, unless the regulatory framework is adjusted to clarify liability issues. ${ }^{67}$

Beyond liability issues, another important point is the extent to which blockchainbased transactions can be considered admissible evidence by a court. ${ }^{68}$ An interesting development in this respect is the ruling by China's Supreme Court, in September 2018, that evidence authenticated with blockchain is binding in legal disputes. ${ }^{69}$ While this ruling is an undeniable step forward, it will not solve all issues. Indeed, unless the true identity of the participant in the transaction is identified, which in the case of public blockchains is complicated, courts may have concerns about blockchain-based transactions being admissible evidence.

\section{Cross-Border Data Flows, Data Localization and Data Privacy Issues}

The increased digitization of our economies, fuelled by the rise of the Internet and of digital technologies, such as artificial intelligence (AI), has brought the issue of cross-border data flows to the forefront of trade policy..$^{70}$ Despite the growing importance of data and data flows for economic activity, many countries have

67 Ibid.

68 J. S. Cermeno, 'Blockchain in Financial Services: Regulatory Landscape and Future Challenges for Its Commercial Application’, BBVA Research Paper No 16 (2016).

69 M. Huillet, 'China's Supreme Court Rules That Blockchain Can Legally Authenticate Evidence', Cointelegraph, 7 September 2018.

70 See Chapter 1 in this volume. 
adopted measures that impose requirements or restrictions on data flows. ${ }^{71}$ These requirements can either be explicitly required by law or can be the result of a series of restrictions that make it de facto impossible to transfer data, such as local storage requirements, local processing of the data or government approval to transfer data. Some countries prohibit all data transfers, while others target specific sectors or services. As for barriers to cross-border data flows, they typically involve restrictions on the transfer of personal data to jurisdictions deemed to provide a lower level of data protection, as well as limitations on information that governments consider 'sensitive'. Governments' motivations for putting in place such policies are diverse and include the wish to protect citizens' privacy, ensure access to data for the purposes of law enforcement, and promote the local economy, as well as potential cybersecurity concerns. It is pertinent to ask to what extent blockchain transactions are likely to be affected by such policies.

\section{Data Localization Restrictions Can Impact Blockchain, Although to a Limited Extent}

Because of their distributed nature, blockchains de facto fulfil local storage and local data processing requirements: in a blockchain, all participants in the network have a local copy of the transactions and every fully participating node must process every transaction. Requirements that take the form of government approval to transfer data would however impede the participation of entities or individuals from the countries concerned in cross-border blockchain applications and thereby undermine the potential of this technology to create a global asset web. Hence, although certain types of requirements on data flows may not directly affect them, blockchain applications are not completely immune from restrictions in this area. The crossborder nature of blockchain does require free cross-border data flows. Lack of a common approach on these issues, and the regulatory fragmentation that would result from it, would ultimately impede the development of a technology that holds high promises to facilitate cross-border transactions at a global level. Discussions on this issue are taking place in the context of the WTO joint initiative on e-commerce. ${ }^{72}$ However, at the time of writing, it remained uncertain how potential obligations would shape up, as the position of participants in the initiative differ substantially on the issue of data flows and data localization.

${ }^{71}$ See Chapter 3 in this volume.

${ }^{72}$ See the proposals submitted by Brazil (INF/ECOM/27); Canada (INF/ECOM/34); the European Union (INF/ECOM/22); Republic of Korea (INF/ECOM/31); Japan (INF/ ECOM/2O); Singapore (INF/ECOM/25); Chinese Taipei (INF/ECOM/24); and the United States $(\mathrm{INF} / \mathrm{ECOM} / 23)$. 


\section{Data Privacy}

With the rise of the digital economy, issues related to data privacy have become a key concern. Blockchain opens new opportunities in this respect and is an interesting innovation for personal data management, but it also gives rise to an intense debate regarding the potential non-compliance of blockchain with data protection regulations, in particular the EU's General Data Protection Regulation (GDPR). ${ }^{73}$ The relationship between blockchain and data privacy is, therefore, both promising and challenging.

A BLOCKCHAIN AS A NEW TOOL FOR DATA SOVEREIGNTY AND PROTECTION Blockchain is often presented as an opportunity or catalyst for better personal data protection and new forms of identity management. While in today's world, service providers like Instagram, Snapchat and Facebook control our online identity and use our data without us necessarily knowing it, sometimes even misusing it, ${ }^{74}$ blockchain gives users control over their data, allowing them to manage and share it only with trusted parties. This is often referred to as 'self-sovereign identity', whereby the usage of one's personal data is controlled by the owner of the identity. ${ }^{75}$ Various companies, such as Sovrin, are now offering services leveraging blockchain to allow individuals to collect, hold and choose which identity credentials to use - such as a driver's license or employment credential - without relying on individual siloed databases that manage the access to those credentials. ${ }^{76}$

One must, however, distinguish here between public and consortium/private blockchains. While public blockchains enable the users themselves to implement the principle of 'privacy by design' at an individual level, ${ }^{77}$ consortium/private blockchains provide for this principle at the platform level, as the privacy protection levels are determined by the management of the platform. On such platforms, participants are known and identified, but permissions to read and write some of the data added to the platform can be restricted to certain participants in order to protect confidentiality. Blockchain is thus an interesting innovation for personal data management but it also raises some challenges.

73 Regulation 2016/679 of the European Parliament and of the Council of 27 April 2016 on the Protection of Natural Persons with Regard to the Processing of Personal Data and on the Free Movement of Such Data, and Repealing Directive 95/46/EC [hereinafter: General Data Protection Regulation or GDPR], OJ L [2016] 119/1.

74 J. Sadowski, ‘Companies Are Making Money from Our Personal Data, But at What Cost?', The Guardian, 31 August 2016.

75 O. Jacobovitz, 'Blockchain for Identity Management', Ben-Gurion University Technical Report o2 (2016); C. Sullivan and E. Burger, 'E-Residency and Blockchain', Computer Law and Security Review 33 (2017), 470-481.

$7^{6}$ https://sovrin.org.

77 A. Biryukov et al., 'Deanonymisation of Clients in Bitcoin $\mathrm{P}_{2} \mathrm{P}$ Network', Cornell University Working Paper (2014). 
B COUld data protection Regulations block blockchain? Data protection legislations have flourished around the world with the objective of giving individuals greater control over the way their data is processed and ensuring that their data is safe and secure. Almost 60 per cent of countries have put in place legislation to secure the protection of data and privacy, and another 10 per cent have draft legislation. ${ }^{78}$ Probably the most well-known of these laws is EU's GDPR, whose entry into force on 25 May 2018 has unleashed heated discussions regarding the possible incompatibility of blockchain with GDPR provisions, leading some to claim that GDPR could 'block' or 'kill' blockchain. ${ }^{79}$

The GDPR applies to the processing of all personal data of data subjects in the European Union, unless data has been anonymized, with personal data defined as 'any information relating to an identified or identifiable natural person'. ${ }^{80}$ The process of anonymization requires not only to make it impossible to identify the person, but it must also be irreversible. ${ }^{81}$ Non-anonymous data, including 'pseudonymous' data, remains subject to the GDPR. The question therefore arises whether blockchain characteristics make it possible to anonymize data, which would exempt blockchain data from the scope of the GDPR. There is an intense debate within the community regarding the various techniques that could be used to anonymize data. The use of asymmetric encryption (private and public key encryption) does not ensure irreversibility. Research has shown that public keys can be traced back to the IP address to de-anonymize users - although the problem is not inherent to the technology and could be addressed by fixing the technical design of the blockchain. ${ }^{82}$ Hashing, which is heavily used in blockchain, offers better prospects, but does not guarantee full anonymization. Although hashing is a non-reversible encryption technique, reversibility and linkability risks can exist under specific circumstances, making it still possible to identify users. ${ }^{83}$ Such risks need to be assessed on a case-by-case basis. ${ }^{8}$ More advanced cryptographic techniques are being developed that can be viable in the mid-term, such as Zero-Knowledge Proof (ZKP), which allows one party to produce a proof of statement without disclosing the data underlying that statement. This method makes it possible,

$7^{8}$ UNCTAD, 'Data Protection and Privacy Legislation Worldwide', available at: https://unctad .org/en/Pages/DTL/STI_and_ICTs/ICT4D-Legislation/eCom-Data-Protection-Laws.aspx.

79 S. Johnson 'Will GDPR Compliance Kill Blockchain?', Medium, 4 July 2018; A. Toth, 'Will GDPR Block Blockchain?', World Economic Forum, 24 May 2018.

8o Article 4(1) GDPR.

${ }^{81}$ T. Lyons, L. Courcelas and K. Timsit, Blockchain and the GDPR (Brussels: European Union Blockchain Observatory and Forum, 2018).

82 Biryukov et al., note 79 .

${ }^{8}$ A reversibility risk could exist, for example, if the original data is of a known and relatively small size (although some techniques exist to mitigate this risk). A linkability risk can exist if the recorded hash is the same every time because a given user orders a transaction, making it possible to analyze times and frequency and to uncover personal data. See Lyons et al., note 83 .

84 Ibid. 
for example, to prove that person $\mathrm{X}$ lives in Geneva without disclosing their exact address.

Two key provisions of the GDPR seem a priori incompatible with blockchain, namely the 'right to rectification' and the 'right to be forgotten' - i.e., the right to rectify or obtain the erasure of personal data. ${ }^{85}$ Indeed, the quasi-immutable nature of blockchains makes it very difficult to update, erase, change or correct data. The GDPR, however, does not specify what constitutes erasure. Some in the community argue that a possible solution is to keep personal data off the chain, with only its evidence (cryptographic hash) exposed to the chain, thereby maintaining the integrity of the transaction while making it possible to erase the transaction itself. The deletion of the data stored externally would mean that the hash stored on the blockchain would point to a location that has been deleted. In addition, in a report published in September 2018, the French National Commission on Informatics and Liberty (CNIL) noted that some encryption techniques, coupled with key destruction, can potentially be considered erasure, 'without resulting in strictly identical effects'. ${ }^{86}$

Beyond these two most well-known and emblematic provisions of the GDPR, other GDPR provisions stand in tension with the way blockchains operate. Indeed, whereas the GDPR was designed for a world where data is centrally collected, stored and processed, blockchains decentralize these processes. ${ }^{87}$ Under the GDPR 'data controllers' (the party that determines the purposes and means of processing particular personal data) and 'processors' (party responsible for processing personal data on behalf of the controller, such as an outsourced provider) have distinct obligations. Determining "who is what" is necessary to assess obligations but can be challenging in a blockchain context.

Data controllers have obligations to process personal data lawfully or face stiff consequences that can be fines as high as EUR 20 million or 4 per cent of a company's worldwide annual turnover, ${ }^{88}$ and they should do everything to ensure that the data is secure. They also have obligations in terms of where the data processing takes place. Under the GDPR, personal data can only be transferred to third countries if they are deemed to provide data protection that is 'adequate' or equivalent to that in the $\mathrm{EU}$, for example, if the organization receiving the data is covered by an agreement The ECJ declared this agreement invalid in July 2020 or where bespoke contractual protections are put in place, such as the EU's 'model clauses'. Whereas identifying the controller and processor is relatively easy in traditional cloud computing systems -

${ }^{85}$ Articles 16 and 17 GDPR.

86 CNIL, 'Blockchain et RGPD: quelles solutions pour un usage responsable en présence de données personnelles ?', Commission Nationale de l'Informatique et des Libertés (CNIL), 24 September 2018.

${ }_{7}$ M. Finck, Blockchain Regulation and Governance in Europe (Cambridge: Cambridge University Press, 2019).

${ }^{88}$ Article 83(5) GDPR. 
typically those uploading personal data to the cloud environment are the controllers and the operators of the cloud system are the processor - the collective processing of data in the context of blockchains makes it difficult to define whether the users are controllers or processors. This is particularly true for public permissionless blockchains. ${ }^{89}$ While it is generally admitted that protocol developers should not be considered data controllers because they simply created the tool, there is a debate regarding validating or participating nodes ${ }^{9 \circ}$ and smart contract users. As for network users, it is generally admitted that if they submit personal data as part of a business activity, they could be considered data controllers, but if they submit their own personal data for their own personal use, they are likely to fall under the household exemption of the GDPR..$^{91}$ The debate has not been settled yet, which has implications for other rights of data subjects under the GDPR, in particular the right of access - i.e., the right for users to enquire of a data controller if their personal data is being processed and if it is, to receive certain details about how this is being done. ${ }^{92}$ If the controller is not identified, users cannot properly exercise their rights.

Blockchain GDPR compliance issues are critically important both because of the extraterritorial nature of the GDPR, ${ }^{33}$ but also because of the cross-border nature of most blockchain platforms. Interestingly, while blockchains and the GDPR seem incompatible at a conceptual level, both pursue the same goal of giving individuals more control over their personal data, but through different mechanisms. Some have argued that consideration could be given to whether the GDPR's underlying objectives could be achieved through means other than those originally envisaged to avoid asphyxiating the development of a technology that holds great promises. ${ }^{94}$ CNIL, who was one of the first authorities to officially address the matter, announced that it would work cooperatively with other European data protection

${ }^{89}$ In the case of private/consortium blockchains, controllers can be identified as part of the governance design structure of the blockchain platform. CNIL actually recommends that blockchain consortiums identify the controller or joint controllers early on in the project.

$9 \circ$ Some argue that these nodes are simply running the protocol in the hope of winning a reward, and that they do not determine the purpose of means of processing. Others, however, note that they should be considered controllers because they are actively running the software and may influence how the platform evolves, for example by choosing - or not - to run a new version of a protocol that is being released.

${ }^{91}$ Lyons et al., note 83 .

$9^{2}$ Article 15 GDPR.

93 A non-EU organization can fall in the scope of the GDPR if it is offering goods or services to individuals in the EU. A Canadian web shop with a website in French and English that processes multiple orders a day from individuals in the EU and ships to the EU would fall in the scope of the GDPR, even though that web shop has no establishment in the EU and is not performing any data processing activities within the EU. Whether the services offered are paid or for free does not matter. In other words, a Canadian free cloud storage service must comply with all the obligations of the GDPR, if the service is also offered to users within the EU.

94 M. Finck, 'Blockchains and Data Protection in the European Union', European Data Protection Law Review 4 (2018), 17-35. 
authorities 'to suggest a strong and harmonized approach'.95 It may also be worth noting that blockchain's built-in tracking and auditability functions could help organizations comply more easily with other GDPR provisions on internal recordkeeping requirements. ${ }^{96}$

While the GDPR only has limited direct relevance to international trade in goods, as most information contained in trade documents relates to companies, not individuals, it could nevertheless impact trade in specific situations, when the contact details of a person at a firm need to be given (e.g., for exports of dangerous goods). Ultimately, the need to find a compromise between ensuring legal protection of personal data and encouraging innovation is one issue that regulators need to grapple with, as the current discussions in the European Union but also on trade negotiation tables show. ${ }^{97}$

\section{DEVISING A WAY FORWARD}

Blockchain is a promising technology whose impact on international trade could be multifaceted and significant. While the years immediately following the release of the first distributed ledger technology have been years of exploration through proofs of concepts, many of which did not go beyond the concept stage, projects have now started to move into production. Gartner predicts that the phase of irrational exuberance, few high profile successes' that we have experienced, will be followed between 2022 and 2026 by a phase of 'larger focused investments, many successful models', and that after 2026 the technology will be a 'global large-scale economic value-add', which could deliver US\$30 trillion of value worldwide by $2030.9^{8} \mathrm{Given}$ the potentially significant impact that the technology could have on economic activity and international trade, it is important that regulators start thinking about the practical and legal implications of blockchain on international trade and ways to support the deployment of the technology while preserving their legitimate right to regulate.

This section discusses the need for the creation of a regulatory environment conducive to the development of the technology through polycentric co-regulation. It then proposes various actions that could be taken at the level of the WTO.

\section{The Need for a Conducive Regulatory Environment}

While regulating too early is not desirable as it could stifle the development of a technology that is still maturing - or worse, fail to adequately regulate its use -

95 CNIL, note 88.

96 Ibid.

97 Ganne, note 8. See also Chapter 9 in this volume.

$9^{8}$ R. Kandaswamy and D. Furlonger, 'Blockchain-Based Transformation', A Gartner Trend Insights Report, 27 March 2018. 
legislators cannot afford to do nothing in the face of the rapid changes that are under way. Regulation can be important, if not indispensable, for the large-scale deployment of the technology.

\section{Regulation as an Enabler}

Technology and regulation are often 'posed as adversaries'. ${ }^{99}$ Yet, technology and innovation need regulation to thrive. The history of the Internet shows that companies might eventually welcome regulation, as it allows them to operate in a more predictable environment and to build consumer confidence. ${ }^{100}$ Code needs to be legally recognized to build value and trust. ${ }^{101}$ This holds true for blockchain as well. Blockchain has the potential to truly digitize trade transactions, but it is only a tool. As seen in the previous sections, without a regulatory framework that provides, for example, for the legal recognition of e-signatures, e-documents and blockchain transactions, and that clarifies liability issues, digitization of trade will remain wishful thinking and technology adoption will not occur.

Legal certainty not only allows stakeholders to evolve within a more predictable environment and gives them tools to achieve what they are thriving for, it also stimulates innovation. The Porter hypothesis, formulated by the economist Michael Porter in 1995, suggests that strict environmental regulations induce efficiency and encourage innovations that help improve commercial competitiveness. Yet, overregulating would be counterproductive and would asphyxiate innovation. Striking the right balance is critical.

\section{The Challenge of Blockchain Regulation}

The rise of the Internet challenged regulators to think out of the box and to devise new regulatory approaches. Regulating blockchain is likely to be equally, if not more, challenging, given the intrinsic characteristics of the technology. ${ }^{102}$ First, blockchain is inherently transnational by nature, which means that unilateral action anchored in territorial jurisdiction makes little sense and could, in the absence of global coordination, lead to damaging regulatory fragmentation. Second, blockchains are decentralized networks that function on a peer-to-peer basis, rendering their evolution hard to predict. This is different from the Internet, which although with a distributed architecture has physical elements - in particular the regulatory

99 Finck, note 96.

${ }^{100}$ J. Wiener, 'The Regulation of Technology, and the Technology of Regulation', Technology in

Society 26 (2004), 483-500.

${ }^{101}$ Finck, note 96.

${ }^{102}$ Ibid. 
access points are centralized, making its regulation possible. ${ }^{103}$ Third, in a traditional environment the decentralized and distributed nature of blockchain, combined with its high level of security and immutability, allow actors who would not transact directly because of lack of trust, to interact on a peer-to-peer basis. This is particularly important in the case of international trade where transactions involve dozens of actors along the supply chain who usually hold their own registries and follow their own processes. Blockchain has the potential to break these sectoral silos but will only be able to do so if regulation is developed at a cross-sectoral level. As noted earlier, various consortia have emerged to facilitate trade finance, transportation and logistics. However, these platforms follow their own logic and, for the time being, do not talk to each other - be it at the technological level, or at the level of semantics, data models and processes. ${ }^{104}$ Fourth, public blockchains are built on the premise of greater anonymity and last but not least, the world of blockchain is a multifaceted and fast evolving world. It is therefore critical that regulators proactively educate themselves, closely follow developments and work with the private sector to devise collective solutions to build a regulatory environment that promotes the technology rather than impedes it. Critically, regulating blockchain does not mean regulating the technology itself but rather its specific use cases.

Regulatory approaches followed so far vary widely across jurisdictions, not only between national jurisdictions, but sometimes even between federal and state jurisdictions, as in the case of the United States. Many jurisdictions have opted for a wait-and-see approach to allow time to observe developments, with some taking a proactive observatory approach. This is the case of the European Union, which launched the EU Blockchain Observatory Forum in February 2018 to actively monitor developments, collect use cases and consult with experts and practitioners in the field before developing specific policies. ${ }^{105}$ Some have chosen to issue guidance, such as the guidelines on Initial Coin Offerings (ICO) published by Switzerland, ${ }^{106}$ while others have developed new legislation, either in the form of amendments to existing laws or standalone legislation, such as the Liechtenstein 'Tokens and TT Service Providers Law', also referred to as Blockchain Act, which entered into force in January 2020.

Various jurisdictions have also launched regulatory 'sandboxes', i.e. governmentbacked initiatives that allow live time-bound testing of innovations under a regulator's oversight. Regulatory sandboxes aim at testing and encouraging innovation by minimizing legal uncertainty while allowing regulators to stay abreast of new business ideas and products, and to learn where they might need to update or fill

\footnotetext{
${ }^{103}$ A. Guadamuz, Networks, Complexity and Internet Regulation: Scale-Free Law (Cheltenham: Edward Elgar, 2011), at 89.

${ }^{104}$ For more information on this issue, see Ganne, note 8.

105 www.eublockchainforum.eu/.

106 'FINMA Publishes ICO Guidelines', Swiss Financial Market Supervisory Authority (FINMA), 16 February 2018.
} 
in gaps in existing regulatory frameworks. Typical features of regulatory sandboxes include customized rules for each firm/business proposal, rather than a one-size-fitsall approach; a limited number of customers/clients, testing for a limited time period, and safeguards for consumer protection (such as requirements to obtain informed consent); restricted authorization/licensing, individual guidance, waivers/ modifications to rules for that project, and no enforcement action letters. ${ }^{107}$ The UK Financial Conduct Authority (FCA) introduced the first regulatory sandbox specific to blockchain in $2016 .{ }^{108}$ Other have followed the UK approach: among them are Australia, Canada, Denmark, Honk Kong, China, Malaysia, South Africa, Switzerland, Chinese Taipei, and more recently Brazil. ${ }^{109}$ While regulatory sandboxes break new ground, one of their key drawbacks is their limited jurisdictional scope. Of greater interest would be the creation of a multi-jurisdictional regulatory sandbox. This is what the Global Financial Innovation Network (GFIN), which was formally launched in January 2019 , aims to do. ${ }^{110}$

Finally, some jurisdictions have chosen the path of regulatory cooperation. ${ }^{111}$ Singapore regulators are working with the Hong Kong Monetary Authority to develop a transnational blockchain-based trade finance system. ${ }^{112}$ Twenty-one EU member states and Norway have signed a declaration on the establishment of a European Blockchain Partnership in April 2018, in the context of which they agreed to cooperate closely to prevent fragmented approaches, and ensure interoperability and wider deployment of blockchain-based services. ${ }^{113}$

While testing and flexibility are important, in particular in the early days of technological innovation, so is some degree of regulatory convergence as the technology matures and projects move into the production phase. The transnational nature of blockchain means that regulatory action cannot be confined to the national level. When it comes to international trade, its potential cross-sectoral impact means that it cannot be confined to certain sectors either. A transnational, trans-sectoral approach is necessary.

${ }^{107}$ K. Agarwal, 'Playing in the Regulatory Sandbox', New York University Journal of Law and Business Online, 8 January 2018.

108 'Regulatory Sandbox', Financial Conduct Authority (FCA), 11 May 2015, available at www.fca .org.uk/firms/regulatory-sandbox.

109 D. Aguilar, 'Brazilian Financial Authorities Announce Regulatory Sandbox for Blockchain', CoinDesk, 17 June 2019, available at www.coindesk.com/brazil-financial-authorities-announceregulatory-sandbox-for-blockchain.

110 The GFIN is a network of thirty-eight financial regulators and related organizations. See 'Global Financial Innovation Network (GFIN)', Financial Conduct Authority (FCA), 31 January 2019, available at www.fca.org.uk/firms/global-financial-innovation-network.

${ }^{111}$ Finck, note 96.

${ }^{112}$ E. Barreto, 'Hong Kong, Singapore to Link Up Trade Finance Blockchain Platforms', Reuters, 25 October 2017.

${ }^{113}$ European Commission, 'European Countries Join Blockchain Partnership', Press Release, 10 April 2018. 


\section{The Need for Blockchain Polycentric Governance}

Because of its decentralized and distributed nature, blockchain requires a matching decentralized and distributed governance system, which some experts call polycentric co-regulation ${ }^{114}$ or polycentric governance. ${ }^{115}$ This is a system whereby regulation is entrusted to parties which are recognized in the field and relies on the fragmentation of authority and power sharing. ${ }^{116}$

The multi-stakeholder approach that governs the Internet provides an interesting model of polycentric governance that could serve as an inspiration for blockchain governance. Internet governance relies on a series of 'global governance networks' that bring together companies, civil society organizations, software developers, academics and governments and that operates on consensus. ${ }^{117}$ These networks include Standards networks, which are non-state, non-profit organizations in charge of developing technical specifications and standards; knowledge networks that conduct research and propose new ideas to help solve global problems; delivery networks, such as the Internet Corporation for Assigned Names and Numbers (ICANN), which is a public-private partnership that delivers Internet domain names and is dedicated to preserving the operational stability of the Internet; policy networks that inform the policy debate and support policy development; advocacy networks that seek to influence the agenda or policies of governments, corporations and other institutions; watchdog networks; and networked institutions, such as the Internet Society, which defines itself as a 'global cause-driven' organization dedicated to ensuring that the Internet remains 'open, globally connected and secure'. ${ }^{118}$

Some networks have started to emerge in the blockchain space, such as the Blockchain Research Institute, ${ }^{119}$ the Blockchain Interoperability Alliance, ${ }^{120}$ and the International Association for Trusted Blockchain Applications (INATBA). ${ }^{121}$ But much remains to be done to put in place a proper governance system that would bring together companies, civil society organizations, software developers, academics, think-tanks, governments and international organizations in various

${ }^{114}$ Finck, note 96.

${ }^{115}$ S. Shackelford et al., 'When Toasters Attack: A Polycentric Approach to Enhancing the Security of Things', University of Illinois Law Review 2 (2017), 415-475, at 439.

${ }^{116}$ See Chapter 4 in this volume.

${ }^{117}$ D. Tapscott and A. Tapscott, 'Realizing the Potential of Blockchain: A Multistakeholder Approach to the Stewardship of Blockchain and Cryptocurrencies', World Economic Forum White Paper, June 2017.

${ }^{118}$ See www.internetsociety.org/.

${ }^{119}$ www.blockchainresearchinstitute.org/.

${ }^{120}$ S. Higgins, 'New Alliance Sets Out to Boost Blockchain Interoperability', CoinDesk, 28 November 2017, available at www.coindesk.com/new-alliance-sets-out-to-boost-blockchaininteroperability.

${ }^{121}$ https://inatba.org/. 
configurations in an effort to develop collective solutions to existing challenges and thereby support the large-scale deployment of the technology.

\section{What Role for the WTO?}

Because it is the only global body that deals with all aspects of international trade, the WTO is uniquely positioned to promote and contribute to the development of a 'trade-enabling' regulatory framework for blockchain. Some issues of direct relevance to blockchain are already being discussed at the WTO in the context of the Joint Statement initiative on electronic commerce, in particular the recognition of e-signatures, e-documents, as well as the question of cross-border data flows. However, more could be done to specifically address the needs of the blockchain space in relation to international trade. In particular, the WTO could choose to actively monitor developments in that sphere, foster multi-stakeholder cooperation and governance, and promote regulatory advances.

\section{Monitoring Blockchain Developments Related to International Trade}

The world of blockchain is evolving extremely fast. One of the challenges for regulators is to keep abreast of developments, be they at the legislative level or at the level of applications for international trade. Fostering transparency of WTO members' trade regime lies at the heart of the WTO work and over the years has become an increasingly important feature of the global trading system. ${ }^{122}$ Monitoring of legislative developments related to blockchain could be performed as part of the WTO Trade Policy Review process to keep track of the evolution of the blockchain regulatory environment at the national level. In addition, standalone reports that would provide regular updates on latest developments at the level of applications (creation of blockchain consortia, developments in trade finance, etc.) would allow regulators to get a better understanding of the scope of the changes. Such reports could be prepared in the context of the WTO committees work or as part of the research function of the WTO. Closely monitoring developments at these two levels would help trade officials build expertise in an area that remains very complex for many to apprehend.

${ }^{122}$ All WTO agreements contains provisions on transparency and members have called for enhanced transparency provisions in virtually every negotiation held since the establishment of the WTO. Transparency issues are also a central feature of current discussions on WTO reform. Transparency goes hand in hand with monitoring of trade policies. The monitoring function of the WTO has evolved significantly since the 1980s. The original 'regular and systematic review of developments in the trading system' via Secretariat notes was replaced by the Trade Policy Review Mechanism, and a new trade monitoring mechanism was put in place following the 2008 global financial crisis to counter off protectionist pressure and ensure adherence to WTO rules. See P. Pedersen et al., 'WTO Trade Monitoring Ten Years on Lessons Learned and Challenges Ahead', WTO Staff Working Paper No o7 (2018). 


\section{Fostering Multi-stakeholder Cooperation and Governance}

Given the transformative impact that blockchain technology could have on international trade and its transnational and trans-sectoral nature, fostering a multistakeholders' dialogue that brings together companies, governments and international organizations, as well as civil society organizations, academics and thinktanks to try and develop collective solutions to existing challenges is of paramount importance. ${ }^{123}$ Being a global player on international trade, the WTO could be a catalyst for such a dialogue on trade issues. It could play the role of convener or facilitator on issues related to international trade with a view to promoting a coordinated approach for blockchain and global trade. The creation of a WTO Global Trade and Blockchain Forum ${ }^{124}$ that brings together representatives from the private sector, governments, civil society organizations and international organizations working on trade and blockchain issues is a step in that direction.

Greater coordination among international organizations working on trade-related blockchain projects would also be welcome. Virtually all international organizations are conducting work on blockchain, often in a siloed manner. Discussions are taking place at the WCO and UN/CEFACT to look into the potential of the technology for border procedures and trade facilitation. Both the ISO and the ITU have put in place working groups to discuss issues related to definitions and standards, as noted earlier. The Organisation for Economic Co-operation and Development (OECD) created a Blockchain Knowledge Center, and the World Bank is involved in various blockchain projects with the support of their Blockchain Center. In April 2020, the International Chamber of Commerce launched Digital Standards Initiative (DSI) with the support of Enterprise Singapore and the Asian Development Bank to develop digital standards to establish a globally harmonized, digitized trade environment. ${ }^{125}$ Different UN organizations are also working on various blockchain projects. To promote synergies and ensure a minimum level of coordination between the various initiatives taken at an international level, an informal expert group composed of high-level officials of the various international organizations working on blockchain projects could be established - along the lines of the WTO Expert Group on Trade Finance that meets once a year.

\section{Promoting a Conducive Regulatory Environment}

Finally, various actions could be taken at a regulatory level to foster the move to paperless trade. References to the UNCITRAL Model Laws on Electronic

${ }^{123}$ Ganne, note 8.

${ }^{124}$ The first edition took place in Geneva on 2 and 3 December 2019.

125 'Digital Trade Standards Initiative launches under the umbrella of ICC', ICC News, 3 April 2020 . 
Transferable Records (2017), on Electronic Commerce (1996, revised in 1998), and on Electronic Signatures (2001), and to the Convention on the Use of Electronic Communications in International Contracts (2005) could be incorporated in WTO law to foster their transposition into national law. As noted earlier, various international organizations, such as ISO and the ITU, are working on developing blockchain standards, including standard definitions. Other organizations, like UN/CEFACT and the ICC DSI, are developing digital standards specifically related to trade. As is the case for TBT and SPS, WTO members could be invited to use such standards when designing national legislation relevant to blockchain and trade. Beyond monitoring through the Trade Policy review mechanism, a more proactive approach could also be followed, whereby WTO members would be encouraged to notify to the WTO any regulatory changes pertinent to blockchain.

The most natural fit for such provisions would, in the current context, be the ongoing Joint Statement Initiative on e-commerce/digital trade, with the obvious drawback that these discussions do not involve all WTO members. ${ }^{126}$ If the political context permits, one could at some point envisage the incorporation of such provisions in a multilateral document, which could take the form of a Code of Good Practice, along the lines of the TBT Code of Good Practice annexed to the TBT Agreement.

\section{E CONCLUSION}

The future of trade depends as much on technological progress as on the way regulation will shape technological innovation. The transnational nature of blockchain is pushing existing boundaries and challenging traditional regulatory approaches. Its global nature requires global regulatory approaches. In a world where people can transact on a peer-to-peer basis across jurisdictions, regulatory action cannot be confined to the national level. Blockchain could have a major impact on international trade. By making it possible to break existing sectoral silos, it could bring trade globalization to another level - provided regulatory action takes place at a cross-sectoral level. A transnational, trans-sectoral approach that involves the various stakeholders involved in international trade, from traders, shippers, banks, government authorities, but also international organizations, academics and civil society organizations, is necessary for blockchain's full potential to be realized.

The WTO is uniquely positioned to foster and contribute to this multi-stakeholders' dialogue. It can help raise awareness and understanding of the technology by monitoring blockchain developments and can play a pivotal role in promoting the

${ }^{126}$ As of March 2019, seventy-seven WTO members have joined the e-commerce initiative. 
development of a conducive regulatory and governance framework to support the large-scale deployment of a technology that holds high promises to truly transform international trade. Where the blockchain adventure will ultimately take us is difficult to predict, but one thing is certain: regulation will play a key role in shaping the outcome. 